\title{
Editorial
}

\section{Nodding Disease or syndrome: What is the way forward?}

\author{
Ndeezi G
}

\begin{abstract}
Department of Paediatrics and Child Health, College of Health Sciences, Makerere University African Health Sciences 2012; 12(3): 240 - 241 http://dx.doi.org/10.4314/ahs.v12i3.24
\end{abstract}

Nodding disease or syndrome is one of those conditions that recently appeared to be of public health concern in some countries in Africa. The disease has been reported to occur in South Sudan, Tanzania, northern Uganda and Liberia. In South Sudan the disease is mainly concentrated along Yei River $^{1,2}$.

Nodding syndrome is a mentally and physically disabling disease that affects children ${ }^{3}$, typically between the ages of 5 and 15. The disease is characterized by episodes of repetitive nodding of the head, with progression to generalized tonicclonic seizures, mental deterioration and physical incapacity ${ }^{1,3}$. During seizures the children lose consciousness and they may fall into fire or hurt themselves. They usually drop out of school because of deterioration in cognitive function and inability to cope with the school environment.

A disease similar to nodding syndrome was first reported in Uganda by Raper ${ }^{4}$ in 1950. This disease was called 'nakalanga' because of the pathological dwarfism and the affected persons were incapable of procreation. It occurred among the dwellers of Mabira forest near the source of the Nile in Uganda. The forest was heavily infested by the black fly. The disease occurred among any of the tribes living in the area provided they had lived in the forested area for a sufficient number of years. The children born by uneventful pregnancies became thin, weak and listless around the time of weaning with progressive mental and physical retardation with maximal severity occurring around 8 to 15 years. The local inhabitants, who had long recognized the disease, had incriminated the bites of the black fly. Similarly, a

*Correspondence author:
Dr. Grace Ndeezi
Department of Paediatrics and Child Health
College of Health Sciences
Makerere University
Kampala, Uganda
E-mail: gndeezi@gmail.com

240 high number of patients with epilepsy and retarded growth was reported in an endemic area for onchocerciasis in western Uganda ${ }^{5}$. However, a study that evaluated the role of Onchocerca volvulus in the development of epilepsy in rural Tanzania reported no difference in microfilaria density between participants with epilepsy and those without ${ }^{6}$.

Since 2009, about 4000 cases of nodding disease have been reported in northern Uganda. For over 20 years northern Uganda was a conflict area and most people lived in camps for the internally displaced. Following a cease-fire in 2006, most of the people have returned to their villages and this period has coincided with increased numbers of nodding disease, with some families reporting more than two cases?

In this issue of African Health Sciences, Tumwine and others ${ }^{8}$ report clinical and epidemiological characteristics of nodding syndrome in Mundri county, South Sudan. The study was carried out in poor rural communities that had been characterised by several displacements between 1994 and 1997. The prevalence of Nodding Syndrome in the study areas was $2.1 \%$ and $4.6 \%$ in the endemic communities. Similar to the epidemic in northern Uganda, some families have more than one affected child. The ratio of male to female cases was 1.2. The age at onset of suspected cases ranged from 2 to 18 years, with a median of 12 years, and the mean duration of illness had been reported to be 3.0 years. Previous case-control studies had shown a positive association between cases and infestation with Onchocerca volvulus microfilaria, evidenced by positive skin snips. The nodding was reported to be worse during meals or upon walking up in the morning, and on the traditional carbohydrate based diet compared to the western style diet. For some children, head nodding did not disrupt eating or the ability to follow commands. Episodes of abnormal behaviour associated with shouting, screaming or running were reported. Physical examination revealed stunting of

African Health Sciences Vol 12 No 3 September 2012 
growth, dwarfism, mental retardation, and poor development of secondary sexual characteristics. Electroencephalographic examination was consistent with progressive epileptic encephalopathy. The authors concluded that Nodding Syndrome was strongly associated with Onchocerca volvulus. There was no evidence to suggest an environmental pollutant, chemical agent, or other toxic factor.

The authors recommend larger studies to elucidate the etiology and optimal treatment for these children. It has been proven that symptomatic treatment with anticonvulsants and other supportive measures such as nutrition rehabilitation improves the quality of life, though not a cure. On going studies in northern Uganda speculate that the disease is associated with vitamin B6 deficiency and probably high doses of vitamin B6 may be beneficial to these children. The disease seems to be more prevalent in the poor communities. Improved social economic status, environmental control measures for microfilaria will go along way to reduce morbidity due to nodding syndrome.

\section{References}

1. CDC. Nodding syndrome - South Sudan, 2011; 2012 Jan 27.
2. www.cmaj.ca/lookup/doi/10.1503/ cmaj.090821. [Accessed 30 ${ }^{\text {th }}$ Oct 2012].

3. Edwards J. Nodding disease confounds clinicians. CMAJ. 2012 Aug 7; 184(11):E585-6.

4. Raper AB, Ladkin RG. Endemic dwarfism in Uganda. East Afr Med J. 1950 Sep; 27(9):33959.

5. Ovuga E, Kipp W, Mungherera M, Kasoro S. Epilepsy and retarded growth in a hyperendemic focus of onchocerciasis in rural western Uganda. East Afr Med J. 1992 Oct;69(10):554-6.

6. Konig R, Nassri A, Meindl M, Matuja W, Kidunda AR, Siegmund V, et al. The role of Onchocerca volvulus in the development of epilepsy in a rural area of Tanzania. Parasitology. 2010 Sep;137(10):1559-68.

7. http://www.huffingtonpost.com/2012/07/ $30 /$ u gan d a - m y s t e r y - n o d d in gdisease_n_1719120.html. [Accessed 30 ${ }^{\text {th }}$ Oct 2012].

8. Tumwine JK, Vandemaele K, Chungong S, Richer M, Anker M, Ayana Y, et al. Clinical and epidemiologic characteristics of nodding syndrome in Mundri County, South Sudan African Health Sciences 2012; 12(3): 242 - 248 